\title{
Oxygen-Dependent Lipid Profiles of Three-Dimensional Cultured Human Chondrocytes Revealed by MALDI-MSI
}

\author{
Brenda Bakker, ${ }^{\dagger}$ Gert B. Eijkel, ${ }^{\ddagger}$ Ron M. A. Heeren, ${ }^{\ddagger 0}$ Marcel Karperien, ${ }^{\dagger}$ Janine N. Post, ${ }^{\dagger}$ \\ and Berta Cillero-Pastor*, ${ }^{*}$
}

${ }^{\dagger}$ Developmental BioEngineering, MIRA Institute for Biomedical Technology and Technical Medicine, Faculty of Science and Technology, University of Twente, 7522 NB Enschede, The Netherlands

${ }^{\ddagger}$ The Maastricht Multimodal Molecular Imaging Institute (M4I), Division of Imaging Mass Spectrometry, Maastricht University, 6229 ER Maastricht, The Netherlands

\section{Supporting Information}

ABSTRACT: Articular cartilage is exposed to a gradient of oxygen levels ranging from $5 \%$ at the surface to $1 \%$ in the deepest layers. While most cartilage research is performed in supraphysiological oxygen levels (19-21\%), culturing chondrocytes under hypoxic oxygen levels $(\leq 8 \%)$ promotes the chondrogenic phenotype. Exposure of cells to various oxygen levels alters their lipid metabolism, but detailed studies examining how hypoxia affects lipid metabolism in chondrocytes are lacking. To better understand the chondrocyte's behavior in response to oxygen, we cultured 3D pellets of human primary chondrocytes in normoxia ( $20 \%$ oxygen) and hypoxia ( $2.5 \%$ oxygen) and employed matrix-

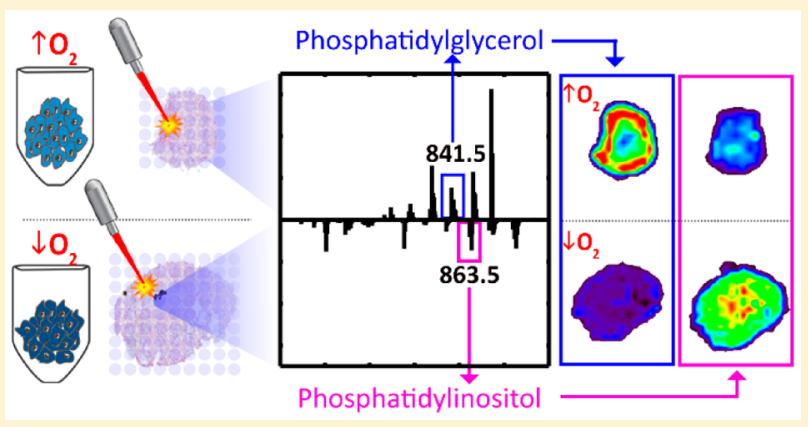
assisted laser desorption ionization mass spectrometry imaging (MALDI-MSI) in order to characterize the lipid profiles and their spatial distribution. In this work we show that chondrocytes cultured in hypoxia and normoxia can be differentiated by their lipid profiles. Among other species, phosphatidylglycerol species were increased in normoxic pellets, whereas phosphatidylinositol species were the most prominent lipids in hypoxic pellets. Moreover, spatial mapping revealed that phospahtidylglyycerol species were less prominent in the center of pellets where the oxygen level is lower. Additional analysis revealed a higher abundance of the mitochondrial-specific lipids, cardiolipins, in normoxic conditions. In conclusion MALDI-MSI described specific lipid profiles that could be used as sensors of oxygen level changes and may especially be relevant for retaining the chondrogenic phenotype, which has important implications for the treatment of bone and cartilage diseases.

A ticular cartilage is an avascular tissue that mainly consists of extracellular matrix (ECM), synthesized by its only residing cell: the chondrocyte. This avascularity results in low oxygen levels of $1-5 \%$ to which chondrocytes are welladapted. $^{1,2}$ In fact, low oxygen levels promote chondrocyte survival in vitro and increase collagen II, aggrecan, and glycosaminoglycan levels. ${ }^{3-7}$ During cartilage degenerative diseases, such as osteoarthritis (OA), the inflammatory processes result in more reactive oxygen species (ROS) $)^{8,9}$ and oxygen levels within the joint are altered. ${ }^{10,11}$

It is well-known that exposure of cells to various oxygen levels alters their lipid metabolism. ${ }^{12-14}$ Lipids exhibit a wide variety of cellular functions, such as structural support, energy storage, protein trafficking, and cell signaling. Recent studies have highlighted the importance of lipid metabolism in cartilage: for example, cholesterol has a regulating role in growth plate chondrogenesis and skeletal development. ${ }^{15,16}$ Moreover, sphingomyelin-related lipids, including acid ceramidase, improve the chondrogenesis of bone marrow-derived mesenchymal stromal cells (MSCs). ${ }^{17,18}$
Moreover, recent studies have shown hypercholesterolemia and altered levels of sphingolipids in the synovial fluid are related to $\mathrm{OA}^{19}$ Lipids also accumulate in articular cartilage during $\mathrm{OA}$, and especially cholesterol and fatty acids are present in the superficial area of OA cartilage. ${ }^{20}$ The World Health Organization estimates that 130 million people worldwide will be afflicted with OA by $2050 .^{21}$ Obesity, a disorder of lipid metabolism, is one of the main risk factors for OA. ${ }^{22}$

Mass spectrometry imaging (MSI) detects thousands of different molecules while preserving their spatial distribution within one tissue section. Once an array of mass spectra is obtained, it is possible to create an image that represents the spatial distribution and relative abundance of that particular molecule, which can then be correlated to histological features, such as cartilage ECM deposition. ${ }^{23}$ The most common MSI methods include time-of-flight secondary ion mass spectrometry (ToF-SIMS), matrix-assisted laser desorption ionization

Received: June 12, 2017

Accepted: July 20, 2017

Published: July 20, 2017 

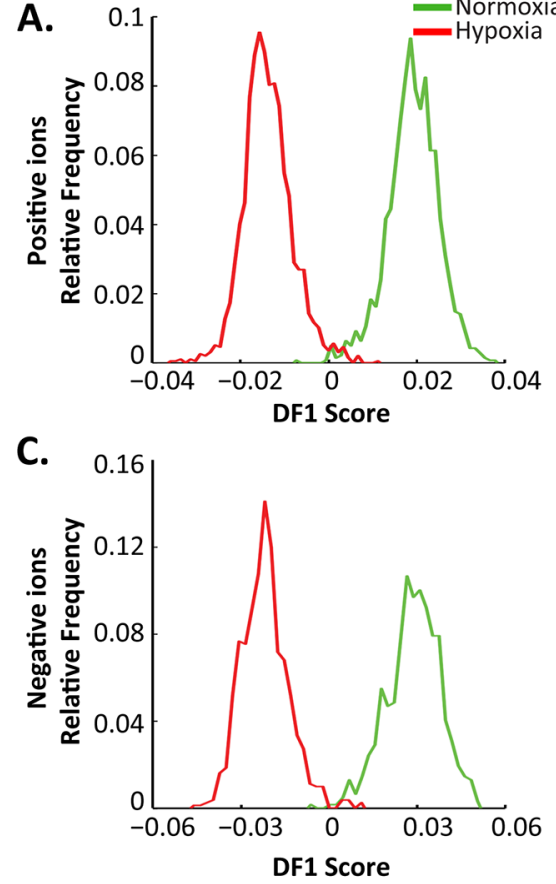

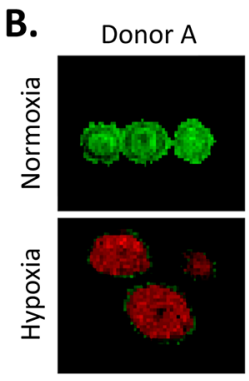

D.

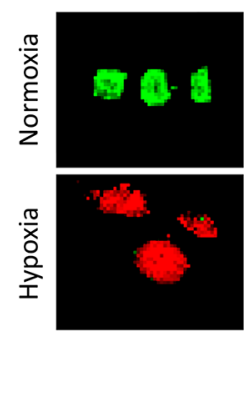

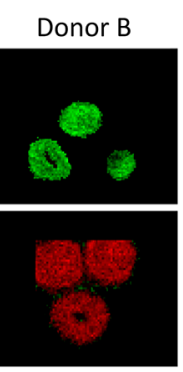
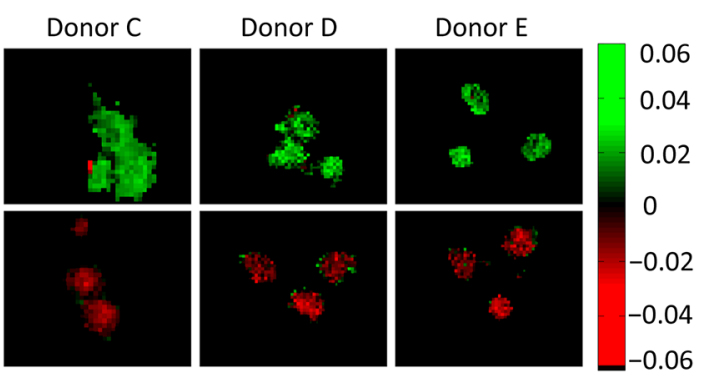

Positive ions - DF1 score projections
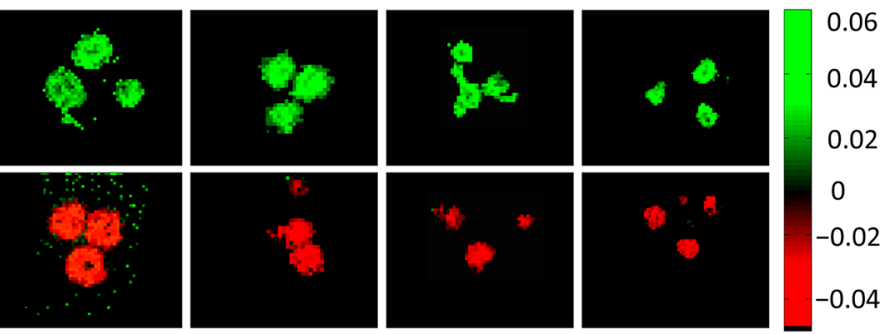

Negative ions - DF1 score projections

Figure 1. Chondrocyte cell pellets cultured in hypoxia $\left(2.5 \% \mathrm{O}_{2}\right)$ and normoxia $\left(20 \% \mathrm{O}_{2}\right)$ were analyzed by MALDI-MSI in negative and positive ion mode. The first discriminant function (DF1) revealed a clear spectral difference between the oxygen levels in positive (A) and negative ion mode (C). The projection of DF1 onto the molecular images showed that the DF1 spectrum was represented by all donors. For both positive (B) and negative (D) ion mode spectra, the score projections describe the DF1+ (green) and DF1- (red) of pellets cultured in normoxia and hypoxia, respectively.

(MALDI), and desorption electrospray ionization (DESI), each with its distinctive capabilities.

Using ToF-SIMS, we previously showed that the lipid profile of chondrogenically differentiated human MSCs, primary chondrocytes, and co-cultures of MSCs and primary chondrocytes are changed in hypoxic cultures $\left(2.5 \% \mathrm{O}_{2}\right)$; in particular, cholesterol was decreased in hypoxic compared to normoxic cultures. ${ }^{13}$

Here, we analyzed the lipid profiles of primary human chondrocytes in $3 \mathrm{D}$ cultures under hypoxic $\left(2.5 \% \mathrm{O}_{2}\right)$ and normoxic $\left(20 \% \mathrm{O}_{2}\right)$ conditions. We employed MALDI-MSI to detect a broader range of molecular species and found distinct lipid profiles for the chondrocytes cultured in different oxygen conditions.

\section{MATERIALS AND METHODS}

Donor Material. Articular cartilage was obtained from five patients (three female and two male) undergoing total knee replacement. The mean patient age was 66 years (range, 53-72 years; Supporting Information Figure S1). Two representative biopsies per patient were used for histological evaluation. The remaining cartilage was used for chondrocyte isolation and subsequent cell culture. The study was approved by the local hospital ethics committee, and all patients gave prior informed consent.

Cartilage Biopsies Evaluation. Two biopsies per patient were formalin-fixed, processed, embedded in paraffin, sectioned, and stained with safranin $\mathrm{O}(0.1 \%$ in water for $10 \mathrm{~min})$ to evaluate the degree of OA-related changes. The degree of cartilage damage was assessed by two independent reviewers according to the OARSI score. ${ }^{24}$ All donor cartilage biopsies showed histological changes, including loss of proteoglycans, discontinuous surfaces, and fissures in the superficial layer to fissures extending into the deep layers (Figure S1). Structural damage and cellular changes were graded using the OARSI score and resulted in an average grade of 3.5 (range, 2.5-4.5).

Cell Culture. Chondrocytes were isolated, as previously described. ${ }^{25}$ In short, cartilage matrix was digested for $20-22 \mathrm{~h}$ in collagenase type II ( $0.15 \%$ Worthington) in DMEM supplemented with penicillin $(100 \mathrm{U} / \mathrm{mL})$ and streptomycin $(100 \mathrm{mg} / \mathrm{mL})$. The digested suspension was filtered through a $100 \mu \mathrm{m}$ mesh nylon filter (cell strainer Nucleon) and washed three times with PBS supplemented with penicillin (100 U/ $\mathrm{mL})$ and streptomycin $(100 \mathrm{mg} / \mathrm{mL})$. Isolated chondrocytes were first expanded in normoxia $\left(20 \% \mathrm{O}_{2}\right)$ or hypoxia $(2.5 \%$ $\mathrm{O}_{2}$ ) until passage 4. Subsequently, high-density micromass cell pellets were formed by seeding 250,000 chondrocytes per well in a round-bottom, low-adherence 96 -well plate and centrifuged for $5 \mathrm{~min}$ at $300 \mathrm{~g}$. Pellet cultures were kept in normoxia $(20 \%$ $\left.\mathrm{O}_{2}\right)$ or hypoxia $\left(2.5 \% \mathrm{O}_{2}\right)$ for 7 days. Chondrocyte proliferation medium (Dulbecco's modified Eagle's medium supplemented with $10 \%$ fetal bovine serum, $1 \times$ nonessential amino acids, 0.2 $\mathrm{mM}$ ascorbic acid 2-phosphate, $0.4 \mathrm{mM}$ proline, $100 \mathrm{U}$ penicillin, and $100 \mathrm{mg} / \mathrm{mL}$ streptomycin) was used for all cultures and was refreshed twice a week.

Oil Red $O$ Staining and Quantification. Pellets embedded in cryomatrix were sectioned and stained with Oil red $\mathrm{O}$ as previously described. ${ }^{13}$ Images were acquired using a Nanozoomer (Hamamatsu Photonics, Hamamatsu, Japan). Lipid droplet accumulation was quantified using Imagej's color threshold function ${ }^{26}$ and revealed a higher abundance of neutral lipids in pellets cultured in normoxia (Figure S2), which is in line with previously reported results. ${ }^{13}$

MALDI-MSI Sample Preparation. Three chondrocyte cell pellets per donor were embedded in $10 \%$ gelatin (SigmaAldrich) in ultrapure water, cut into $10 \mu \mathrm{m}$ thick sections using 
A.

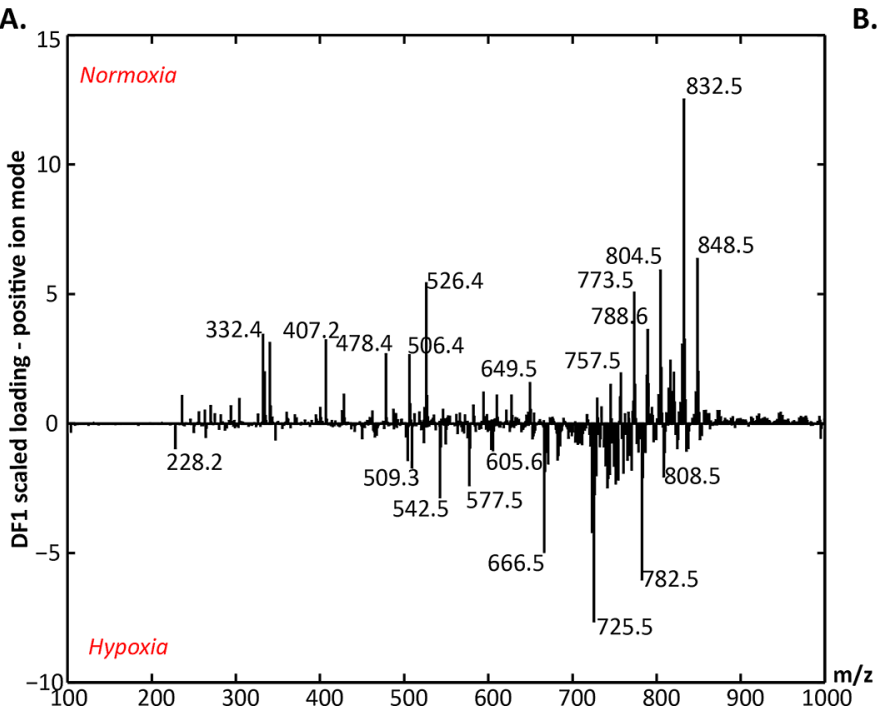

B.

\begin{tabular}{|c|c|c|c|}
\hline $\mathrm{m} / \mathrm{z}$ value & Lipid assignment & Designation & Condition \\
\hline 478.4 & fragment PC $34: 1$ & {$[\mathrm{M}+\mathrm{K}]^{+}$} & Normoxia \\
\hline 506.4 & PC fragment $38: 1$ & {$[\mathrm{M}+\mathrm{H}]^{+}$} & \\
\hline 526.4 & fragment PC $38: 4$ & {$[\mathrm{M}+\mathrm{Na}]^{+}$} & \\
\hline 649.5 & fragment PC 38:4 & {$[\mathrm{M}+\mathrm{Na}]^{+}$} & \\
\hline 757.5 & fragment PC 38:1 & {$[\mathrm{M}+\mathrm{H}]^{+}$} & \\
\hline 773.5 & $\begin{array}{l}\text { fragment PC } 40: 7 \text { or } \\
\text { PC } 38: 4\end{array}$ & $\begin{array}{c}{[\mathrm{M}+\mathrm{H}]+\text { or }} \\
{[\mathrm{M}+\mathrm{Na}]^{+}}\end{array}$ & \\
\hline 788.5 & PC 18:0_18:1 & {$[\mathrm{M}+\mathrm{H}]^{+}$} & \\
\hline 804.5 & PC $36: 4$ & {$[\mathrm{M}+\mathrm{Na}]^{+}$} & \\
\hline 832.5 & PC $38: 4$ & {$[\mathrm{M}+\mathrm{Na}]^{+}$} & \\
\hline 848.5 & PC 38:4 & {$[\mathrm{M}+\mathrm{K}]^{+}$} & \\
\hline 542.5 & fragment SM d18:1/16:0 & {$[\mathrm{M}+\mathrm{Na}]^{+}$} & Hypoxia \\
\hline 577.5 & fragment PC $34: 1$ & {$[\mathrm{M}+\mathrm{Na}]^{+}$} & \\
\hline 666.5 & fragment SM d18:1/16:0 & {$[\mathrm{M}+\mathrm{Na}]^{+}$} & \\
\hline 725.5 & SM d18:1/16:0 & {$[\mathrm{M}+\mathrm{Na}]^{+}$} & \\
\hline 782.5 & PC 34:1 & {$[\mathrm{M}+\mathrm{Na}]^{+}$} & \\
\hline 808.5 & PC 18:0_18:2 & {$[\mathrm{M}+\mathrm{Na}]^{+}$} & 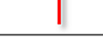 \\
\hline
\end{tabular}

Figure 2. Chondrocyte cell pellets cultured in hypoxia $\left(2.5 \% \mathrm{O}_{2}\right)$ and normoxia $\left(20 \% \mathrm{O}_{2}\right)$ were analyzed with MALDI-MSI in positive ion mode. The average spectra of all donors revealed a clear spectral difference between the oxygen levels in the first discriminant function (DF1) (A). Lipid assignments based on tandem mass spectrometry (MS/MS) identified changes in the phosphatidylcholine (PC) and sphingomyelin (SM) content of the cell pellets (B). The MS/MS spectra defined the fatty acid composition and position as the total of the two chains $\left(\mathrm{sn}_{x+y}\right)$, the individual fatty acids $\left(\mathrm{sn}_{x_{-}} \mathrm{sn}_{y}\right)$ or the individual fatty acids and their respective position $\left(\mathrm{sn}_{1} / \mathrm{sn}_{2}\right)$.

a cryostat (Leica Microsystems), and thaw-mounted on conventional microscope slides. Slides were stored at $-20{ }^{\circ} \mathrm{C}$ until further analysis. Prior to matrix application, slides were placed in a vacuum desiccator at room temperature for $20 \mathrm{~min}$ to be defrosted and dried. For positive ion mode experiments, 20 layers of $5 \mathrm{mg} / \mathrm{mL}$ of $\alpha$-cyano-4-hydroxycinnamic acid (CHCA; Sigma-Aldrich, Munich, Germany) dissolved in methanol/water $(7: 3, \mathrm{v} / \mathrm{v})$ with $0.1 \%$ trifluoroacetic acid were applied. Norharmane, 15 layers of $7 \mathrm{mg} / \mathrm{mL}$ in chloroform/ methanol $(2: 1,(\mathrm{v} / \mathrm{v}))$, was used for the negative ion mode analysis. Cell pellets were homogeneously covered by the matrix solution using the SunCollect spraying device (SunChrom, Friedrichsdorf, Germany).

MALDI-MSI Acquisition. MALDI-MSI experiments were performed on a Synapt G2Si HDMS Q-ToF (Waters Corp., Milford, MA, USA). Spectra were acquired at a raster size of $100 \mu \mathrm{m}$ and in the $m / z$ range of $100-1000$, extended to $m / z$ 2000 for cardiolipin detection. Both positive and negative ion spectra were obtained in "resolution MS mode". The instrument was calibrated using red phosphorus. ${ }^{27}$ Afterward, the MALDI-MSI sections were stained with Alcian Blue to visualize cartilage-matrix deposition.

MALDI-MSI Data Analysis. Principal components analysis (PCA) and linear discriminant analysis (LDA) were used to search for spectral similarities and differences between the samples using an in-house-built ChemomeTricks toolbox for MATLAB version 2012B (The MathWorks, Natick, MA, USA). ${ }^{28}$ First, raw data were converted to a suitable format for BioMap (Novartis, Basel, Switzerland) at a bin size of $\mathrm{m} / z$ 0.05 , followed by conversion to a MATLAB format. Subsequently, in order to only include on-pellet spectra, cell pellet-specific spectra were selected by making an overlay of the MSI with the Alcian Blue image of the same section. The inhouse-developed PEAPI software was used for peak-picking ${ }^{28}$ to reduce the size of the data and to ensure that each mass channel represents a single peak. Finally, LDA was performed by employing the double-stage PCA approach to look for the peaks with the highest differences between normoxia and hypoxia. After the LDA, images were generated by projection of the mass spectra of all $X, Y$ positions (i.e., pixels) on the first discriminant function (DF1). The resulting score for each spectrum was plotted on its original $X, Y$ position (Figure 1B,D). A two-color scale was used to maximize the contrast between the positive and negative scoring spectra. Individual $\mathrm{m} / \mathrm{z}$ values were visualized using BioMap. These intensity images were normalized to the total ion count (TIC) per spectrum to eliminate variations due to ionization efficiency, tissue suppression, or matrix deposition.

Alcian Blue Staining. Following MALDI-MSI, sections were stained for sulfated glycosaminoglycan (GAGs) with Alcian Blue (1\%, in water, pH 1.0 adjusted with $\mathrm{HCl}, 45 \mathrm{~min}$ ) combined with a Nuclear Fast Red counterstain (0.1\% in 5\% aluminum sulfate, $5 \mathrm{~min}$ ).

Lipid Identification. Lipid identification was performed with tandem mass spectrometry (MS/MS) in both positive and negative ion modes using a Synapt G2Si HDMS Q-ToF (Waters) after completing MALDI-MSI experiments on adjacent sections. Lipid ion precursors were selected within a $1 \mathrm{Da}$ selection window and fragmented using 20-30 V collision energy applied in the trap cell. The obtained spectra were processed using MassLynx software (Waters). The MS/MS fragmentation pattern from each selected mass was used to identify the lipids of interest and to assign them to previously classified lipid identifications in the LIPID Metabolites and Pathways Strategy Structure Database (LMSD; www.lipidmaps. org).

\section{RESULTS}

We performed MALDI-MSI on cryosections of chondrocyte pellets in both positive and negative ion modes to assess which lipids are altered by oxygen and to visualize their spatial distribution. PCA followed by LDA separated the data sets into two groups that readily distinguished normoxic and hypoxic cultures. For the positive ion spectra, the frequency plot of the DF1 shows a clear separation between normoxic and hypoxic cultures and describes $2.04 \%$ of the total variance (Figure 1A). 
A.

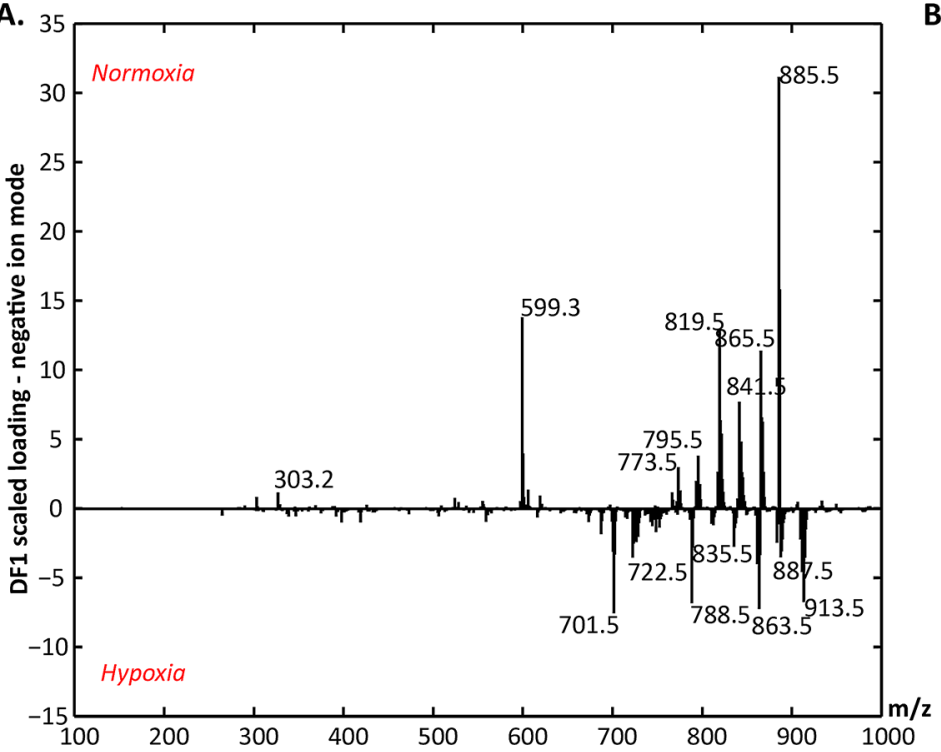

\begin{tabular}{|c|c|c|c|}
\hline $\mathrm{m} / \mathrm{z}$ value & lipid assignment & Designation & Condition \\
\hline 303.2 & Arachidonic acid & {$[\mathrm{M}-\mathrm{H}]^{-}$} & Normoxia \\
\hline 599.3 & fragment PI 38:4 & {$[\mathrm{M}-\mathrm{H}]^{-}$} & \\
\hline 773.5 & PG 18:1/18:1 & {$[\mathrm{M}-\mathrm{H}]^{-}$} & \\
\hline 795.5 & PG 18:1_20:4 & {$[\mathrm{M}-\mathrm{H}]^{-}$} & \\
\hline 819.5 & PG 18:1_22:6 & {$[\mathrm{M}-\mathrm{H}]^{-}$} & \\
\hline 841.5 & PG 20:4_22:6 & {$[\mathrm{M}-\mathrm{H}]^{-}$} & \\
\hline 865.5 & PG 22:6/22:6 & {$[\mathrm{M}-\mathrm{H}]^{-}$} & \\
\hline 885.5 & PI 18:0_20:4 & {$[\mathrm{M}-\mathrm{H}]^{-}$} & \\
\hline 701.5 & fragment PS 36:1 & {$[\mathrm{M}-\mathrm{H}]^{-}$} & Hypoxia \\
\hline 722.5 & PE p36:4 & {$[\mathrm{M}-\mathrm{H}]^{-}$} & \\
\hline 788.5 & PS 18:0_18:1 & {$[\mathrm{M}-\mathrm{H}]^{-}$} & \\
\hline 835.5 & PI 16:0_18:1 & {$[\mathrm{M}-\mathrm{H}]^{-}$} & \\
\hline 863.5 & PI 18:0_18:1 & {$[\mathrm{M}-\mathrm{H}]^{-}$} & \\
\hline 887.5 & PI 18:0_20:3 & {$[\mathrm{M}-\mathrm{H}]^{-}$} & \\
\hline 913.5 & PI 18:0_22:4 & {$[\mathrm{M}-\mathrm{H}]^{-}$} & 1 \\
\hline
\end{tabular}

Figure 3. Chondrocyte cell pellets cultured in hypoxia $\left(2.5 \% \mathrm{O}_{2}\right)$ and normoxia $\left(20 \% \mathrm{O}_{2}\right)$ were analyzed with MALDI-MSI in negative ion mode. The average spectra of all donors revealed a clear spectral difference between the oxygen levels in DF1 (A). Lipid assignments based on tandem mass spectrometry (MS/MS) identified changes in the phosphatidylinositol (PI), phosphatidylglycerol (PG), and phosphatidylserine (PS) content of the cell pellets (B). The MS/MS spectra defined the fatty acid composition and position as the total of the two chains $\left(\mathrm{sn}_{x+y}\right)$, the individual fatty acids $\left(\mathrm{sn}_{x_{-}} \mathrm{sn}_{y}\right)$, or the individual fatty acids and their respective position $\left(\mathrm{sn}_{1} / \mathrm{sn}_{2}\right)$.

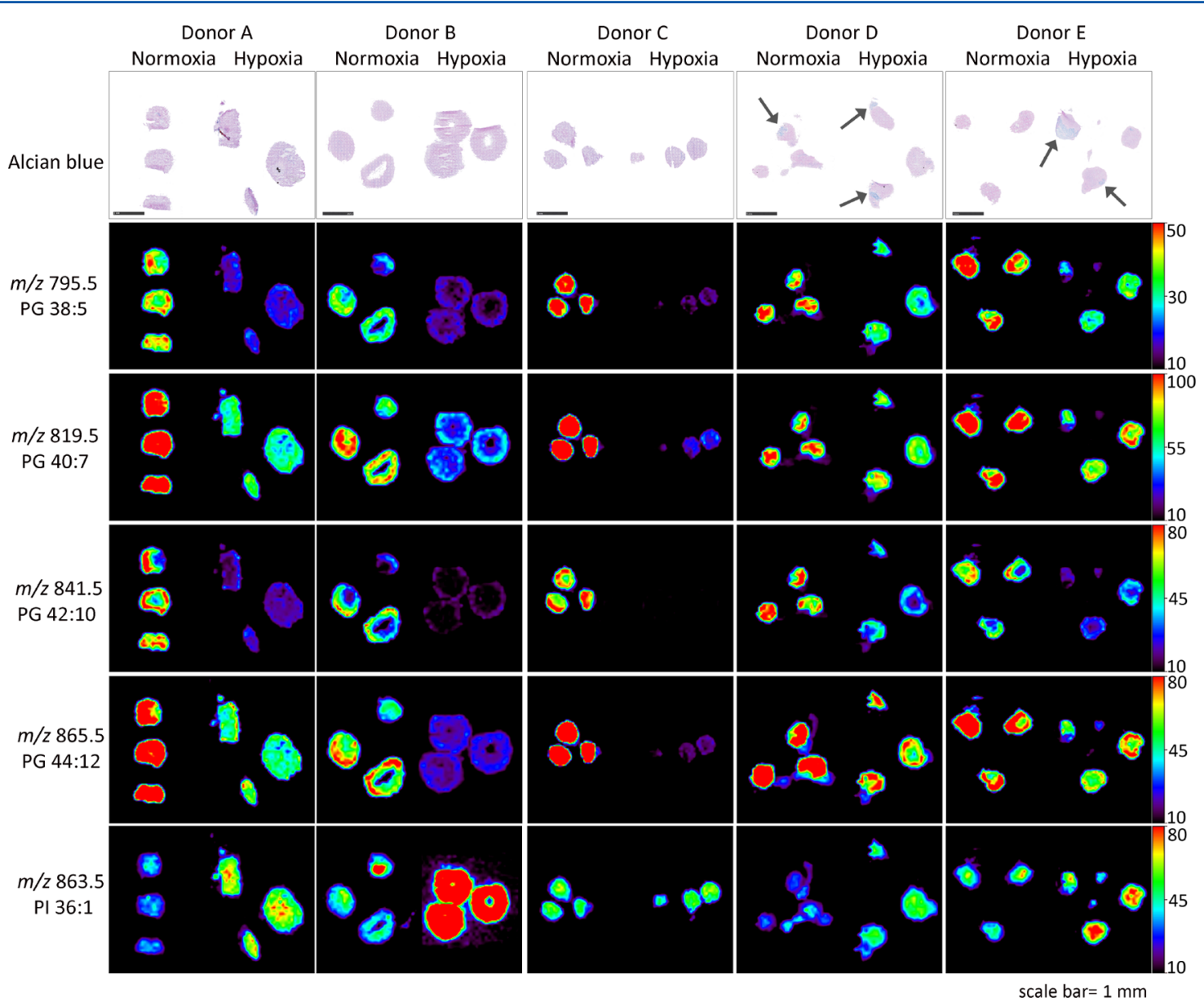

Figure 4. Cartilage-specific extracellular matrix production. After MALDI-MSI, sections were stained with Alcian blue to detect cartilage-specific extracellular matrix production. Arrows indicate clear positive staining. Intensity images of individual PG and PI species show differences between pellets cultured in hypoxia and normoxia. The intensity of the PI at $\mathrm{m} / z 863.5$ (bottom row) is not only higher in hypoxic cultures but also has a higher intensity in the center of normoxic pellets, where the oxygen level generally is lower. The opposite can be seen for the PG at $m / z 841.5$, which is less abundant in hypoxic cultures and in the hypoxic regions (center) of normoxic pellets. 


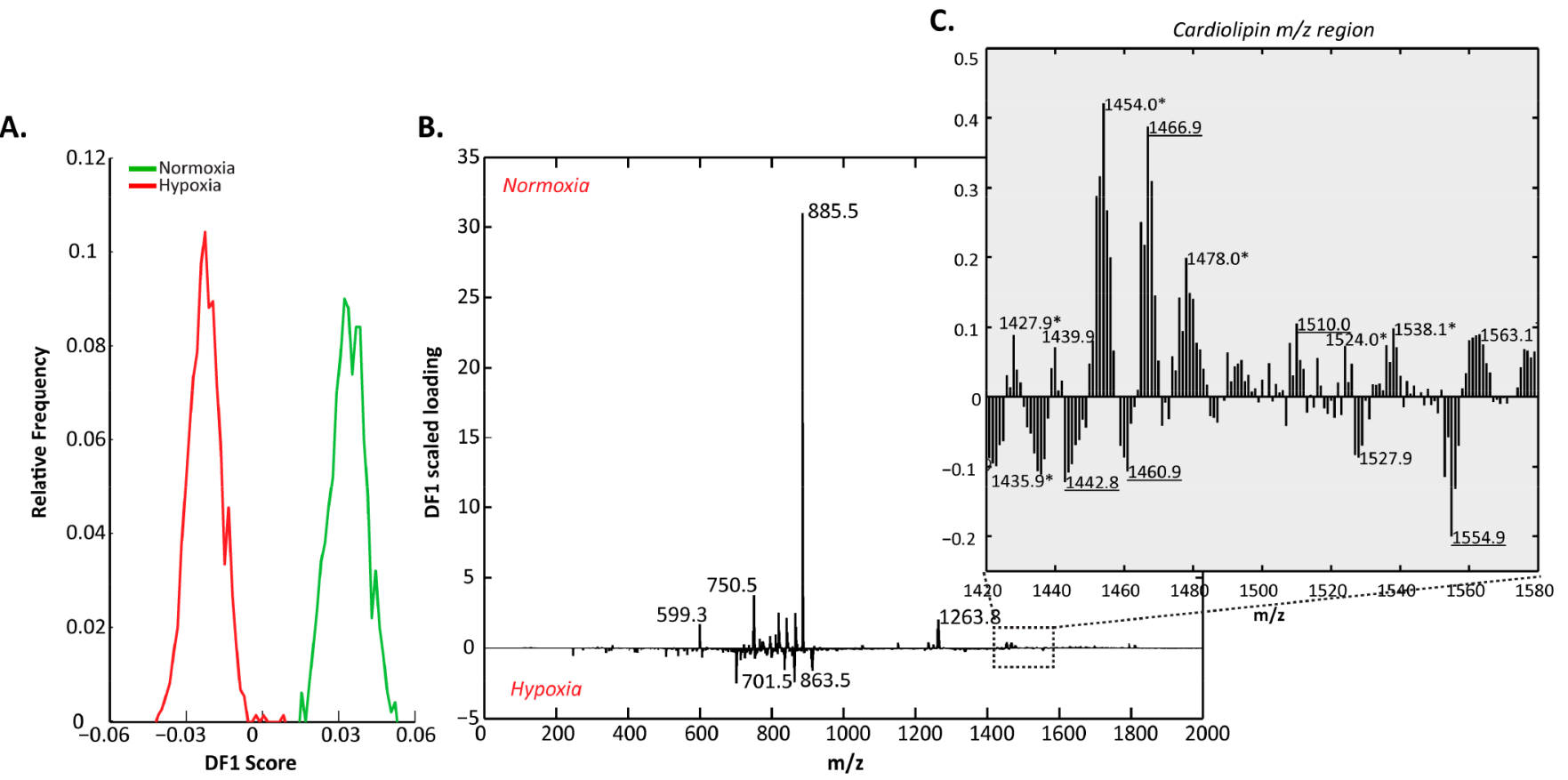

Figure 5. Chondrocyte cell pellets cultured in normoxia $\left(20 \% \mathrm{O}_{2}\right)$ and hypoxia $\left(2.5 \% \mathrm{O}_{2}\right)$ were analyzed with MALDI-MSI in negative ion mode at $\mathrm{m} / z$ 100-2000. (A, B) DF1 revealed a clear spectral difference between the oxygen levels. (C) Enlarging the $\mathrm{m} / z 1420-1580$ revealed more cardiolipin species in normoxia than hypoxia. Asterisked values $(*)$, cardiolipin; underlined values, ganglioside.

Similar to the positive ion spectra, the frequency plot of the DF1 of the negative ion spectra shows a separation between normoxic and hypoxic cultures and describes $6.07 \%$ of the total variance (Figure 1C). DF1 score images were generated to assess the degree to which DF1 spectra are represented by the donors. The DF1+ scores are observed in normoxic pellets and the DF1- scores in hypoxic pellets (Figure 1B,D). The score projections consistently represented the DF1 of their respective culture condition.

Phosphatidylcholine and Sphingomyelin Species Discriminate between Normoxic and Hypoxic Cultures. The discriminant loading spectrum of the positive ions displays specific peaks for normoxic and hypoxic cultures (Figure 2A). The positive fraction represents the molecular profile for normoxic cultures and shows that especially $m / z 526.4,773.5$, $804.5,832.5$, and 848.5 are discriminative for this condition. These peaks were identified with MS/MS as phosphatidylcholine (PC) species 36:4, 38:4, and 38:4 (m/z 804.5, 832.5, and 848.5 , respectively) and fragments of PC $38: 4(\mathrm{~m} / z 526.4)$ (Figure 2B). The negative fraction of the DF1 spectrum represents the molecular profile for hypoxic cultures and shows $m / z 542.5,577.5,666.5,725.5,782.5$, and 808.5 (Figure 2A). These peaks were identified with $\mathrm{MS} / \mathrm{MS}$ as sphingomyelin (SM) d18:1/16:0 $(\mathrm{m} / z$ 725.5), PC species $34: 1$ and $36: 2(\mathrm{~m} / z$ 782.5 and 808.5, respectively), fragments of SM d18:1/16:0 $(\mathrm{m} / z 542.5$ and 666.5$)$, and a fragment of PC $34: 1(\mathrm{~m} / z 577.5)$ (Figure 2B).

Phosphatidylglycerol Species Predominantly Present in Normoxic Cultures. Complementary to the positive ion mode spectra, negative ion mode spectra were acquired on adjacent sections. The discriminant loading spectrum of the negative ions displays specific peaks for normoxic and hypoxic cultures (Figure 3A). The positive fraction represents the molecular profile for normoxic cultures and shows that especially $m / z 599.3,773.5,795.5,819.5,841.5,865.5$, and 885.5 were more abundant in this condition. These peaks were identified with MS/MS as phosphatidylinositol (PI) 38:4 (m/z 885.5), phosphatidylglycerol (PG) 36:2, 38:5, 40:7, 42:10, and $44: 12(\mathrm{~m} / z$ 773.5, 795.5, 819.5, 841.5, and 865.5, respectively), and a fragment of PI 38:4 ( $\mathrm{m} / z$ 599.3) (Figure 3B). The negative fraction of the DF1 spectrum represents the molecular profile for hypoxic cultures and shows that $m / z 701.5,722.5$, 788.5, 835.5, 863.5, 887.5, and 913.5 were the most prominent. MS/MS experiments (Figure 2B) identified PI 34:1, 36:1, 38:3, and 40:4 $(\mathrm{m} / z$ 835.5, 863.5, 887.5, and 913.5, respectively), phosphatidylserine (PS) 36:1 $(\mathrm{m} / z$ 788.5), phosphatidylethanolamine (PE) p36:4 $(m / z 722.5)$, and a fragment of PS 36:1 $(m / z$ 701.5).

Phosphatidylglycerol Species Localized in OxygenRich Areas. As described above, in the negative ion mode spectra PG peaks are, based on their loadings, the most separating masses between oxygen levels. Selected molecular images were reconstructed using BioMap to verify if individual PG and PI species display signal intensity differences between the oxygen levels. These molecular images show that the individual PG masses, including $m / z$ 773.5, 795.5, 819.5, 841.5, and 865.5 , have a higher signal intensity in pellets cultured in normoxia (Figure 4), which is in line with the LDA result. The intensity of the PI at $m / z 863.5$ is not only greater in hypoxic cultures but also has a higher signal in the center of normoxic pellets, where the oxygen level generally is lower. The opposite is the case for the PG at $m / z 841.5$, which is less abundant in hypoxia and in hypoxic regions (center) of normoxic pellets. This shows that normoxia specifically elevates the amount of PG species.

Cardiolipin Species More Abundant in Normoxia. In eukaryotic cells PG is mainly known for its role as precursor for cardiolipin (CL). CL is a unique tetra-acyl phospholipid found in mitochondrial membranes where it plays an important role in mitochondrial bioenergetics, membrane stability, and apoptosis. $^{29-31}$ We analyzed the samples with MALDI-MSI using a higher mass range $(m / z 100-2000)$ to determine 
whether the elevated levels of PG in normoxia result in altered CL composition. LDA revealed a higher contribution of five CL species $(m / z 1427.9,1454.0,1478.0,1524.0$, and 1538.1) in normoxia and only one $(m / z 1435.9)$ in hypoxia (Figure 5$)$. These findings demonstrate that normoxia also results in more abundant CL species compared to hypoxia.

\section{DISCUSSION}

Lipids exhibit a wide variety of cellular functions, such as structural support, energy storage, protein trafficking, and cell signaling. The role of lipids in cartilage degenerative disease, such as $O A$, has gained increasing attention over the years. During OA, the oxygen levels within the joint are altered and oxygen levels have been shown to modulate the lipid content of cells. ${ }^{10,32}$ We therefore set out to study the effect of oxygen levels (hypoxic and normoxic conditions) on the lipid composition of human chondrocytes by employing a widely used in vitro pellet culture system. Using MALDI-MSI and tandem MS, we located the spatial distribution and identified many lipid molecules within the same tissue section from five human donors. LDA showed that human chondrocyte pellets cultured in normoxia and hypoxia have a clear lipid signature, especially in their anionic lipid species.

For hypoxic cultures in positive ion mode, we identified SM d18:1/16:0 as the most discriminative; the sodiated molecular ion $(\mathrm{m} / z 725.5)$ as well as two fragments $(\mathrm{m} / z 542.5$ and 666.5) had a high contribution the DF1-. SM belongs to the class of sphingolipids (SL), and these lipids regulate many processes, including stress responses, proliferation and differentiation, apoptosis, and senescence. ${ }^{33}$ SM 34:1 is the most abundant SM species in synovial fluid and its level is elevated in OA and rheumatoid arthritis. ${ }^{19}$ Rocha et al. showed that SM levels are decreased during chondrogenic differentiation of MSCs, which could be explained by higher gene expression of SPHK $1 .{ }^{18}$ In breast tumor xenografts SM d18:1/16:0 was also more abundant in hypoxic than normoxic areas. ${ }^{34}$

In the negative ion mode we identified the anionic lipid species PI as more abundant in hypoxia and PG as more abundant in normoxia (Figure 2). PI and PG species are biosynthesized by the same central liponucleotide intermediate, cytidine-diphosphate diacylglycerol (CDP-DAG), suggesting competing synthesis. This assumption is corroborated by data obtained in lung surfactant, where a switch in the PI and PG content is observed around the time of birth. In fetal surfactant, PI levels are high while PG is low; this ratio changes around term resulting in high PG and low PI levels in adults. ${ }^{35}$ There is no consensus on why this shift takes place and if and how it is controlled, but it may be regulated in part by the exposure to oxygen. Altered gene expression of the enzymes involved in the formation of PI and PG showed no difference in their expression levels (data not shown), suggesting that this difference occurs at the protein, lipid, or downstream enzyme activity level.

It is unknown if PG has a direct function in the mitochondria of mammalian cells but, as the precursor of CL, may have an indirect role. We found elevated CL species in normoxia compared to hypoxia (Figure 4). CL is a mitochondrial membrane stabilizing phospholipid and plays an important role in mitochondrial bioenergetics and apoptosis. ${ }^{29-31}$ Heywood and Lee showed an increase in the mitochondrial mass in chondrocytes cultured in $20 \%$ oxygen compared to $2 \%$ oxygen. $^{36}$ The accumulation of PG and CL species in chondrocytes in normoxia may thus signify mitochondrial defects, which may explain why chondrocytes perform poorly and lose their phenotype in normoxic conditions.

Another remarkable observation is the length of the acyl chains of the identified PGs. The CL acyl chain length varies in composition in various organs, ${ }^{37}$ but in general the most abundant species is linoleic acid (18:2) followed by oleic acid (18:1). The length of fatty acyl chains and their degree of (un)saturation define the exact function of a phospholipid, and alterations have been associated with disease. For instance, elevated levels of arachidonic (ARA; 20:4) and docosahexaenoic acid (DHA; 22:6) containing CL are linked to cardiomyopathies. ${ }^{38}$ We found, next to the dioleic PG $(\mathrm{m} / z$ 773.5), ARA- and DHA-containing PGs $(m / z$ 795.5, 819.5, 841.5 , and 865.5). Additionally, the five CL species, CL 70:4, $72: 5,74: 7,78: 12$, and $78: 5(\mathrm{~m} / z$ 1427.9, 1454.0, 1478.0, 1524.0, and 1538.1, respectively), that are more abundant in normoxia contain unsaturated acyl chains whereas the CL that is more present in hypoxia, CL 70:0 $(\mathrm{m} / z$ 1435.9), does not contain unsaturated acyl chains. CL is, due to its high level of unsaturation and proximity to ROS production, more prone to peroxidation than other phospholipid classes. The configuration of the acyl chains in CL in healthy cartilage is unknown. Additional studies are needed to determine the role of increased ALA- and DHA-containing PG and CL species in chondrocytes.

The intensity images of PG and PI show a clear location dependency (Figure 4). These images also show variation in intensities between triplicates that may be explained by the location of the section with respect to the spherical pellet, at the edge or more to the middle of the pellet. This implies that the intensities of certain molecules may be used as a marker for its original location within the $3 \mathrm{D}$ tissue section.

Future studies will determine whether these lipid changes also occur in OA affected cartilage in which the oxygen levels are altered and thereby may be used as a marker for disease grade or even therapy.

\section{CONCLUSION}

In conclusion, using MALDI-MSI, we show that human chondrocytes have a distinct, oxygen-dependent lipid profile. These specific lipid profiles may be relevant for retaining the chondrogenic phenotype which has important implications for the treatment of bone and cartilage diseases.

\section{ASSOCIATED CONTENT}

\section{Supporting Information}

The Supporting Information is available free of charge on the ACS Publications website at DOI: 10.1021/acs.analchem.7b02265.

Figure S1 showing the histology of donor site cartilage, Figure S2 showing neutral lipid content, and Figure S3 showing MS/MS fragmentation spectra (PDF)

\section{AUTHOR INFORMATION}

\section{Corresponding Author}

*E-mail: b.cilleropastor@maastrichtuniversity.nl. ORCID -

Ron M. A. Heeren: 0000-0002-6533-7179

Berta Cillero-Pastor: 0000-0002-7407-1165

Notes

The authors declare no competing financial interest. 


\section{ACKNOWLEDGMENTS}

We thank Ufuk Tan Timur for histological assessment of cartilage biopsies and Hang Nguyen for editing. This work is financially supported by the Dutch Arthritis Association (Grant 13-2-402) and the Dutch Province of Limburg as part of the "LINK" program.

\section{REFERENCES}

(1) Silver, I. A.; Maroudas, A. Philos. Trans. R. Soc., B 1975, 271, 261-272.

(2) Zhou, S.; Cui, Z.; Urban, J. P. Arthritis Rheum. 2004, 50, 39153924

(3) Bouaziz, W.; Sigaux, J.; Modrowski, D.; Devignes, C. S.; FunckBrentano, T.; Richette, P.; Ea, H. K.; Provot, S.; Cohen-Solal, M.; Hay, E. Proc. Natl. Acad. Sci. U. S. A. 2016, 113, 5453-5458.

(4) Foldager, C. B.; Nielsen, A. B.; Munir, S.; Ulrich-Vinther, M.; Soballe, K.; Bunger, C.; Lind, M. Acta orthopaedica 2011, 82, 234-240.

(5) Grimshaw, M. J.; Mason, R. M. Osteoarthritis and cartilage/OARS, Osteoarthritis Research Society 2000, 8, 386-392.

(6) Henderson, J. H.; Ginley, N. M.; Caplan, A. I.; Niyibizi, C.; Dennis, J. E. Tissue Eng., Part A 2010, 16, 1585-1593.

(7) Markway, B. D.; Cho, H.; Johnstone, B. Arthritis research \& therapy 2013, 15, R92.

(8) Ziskoven, C.; Jäger, M.; Kircher, J.; Patzer, T.; Bloch, W.; Brixius, K.; Krauspe, R. Can. J. Physiol. Pharmacol. 2011, 89, 455-466.

(9) Lepetsos, P.; Papavassiliou, A. G. Biochim. Biophys. Acta, Mol. Basis Dis. 2016, 1862, 576-591.

(10) Lund-Olesen, K. Arthritis Rheum. 1970, 13, 769-776.

(11) Stevens, C. R; Williams, R. B.; Farrell, A. J.; Blake, D. R. Ann. Rheum. Dis. 1991, 50, 124-132.

(12) Ackerman, D.; Simon, M. C. Trends Cell Biol. 2014, 24, 472478.

(13) Georgi, N.; Cillero-Pastor, B.; Eijkel, G. B.; Periyasamy, P. C.; Kiss, A.; van Blitterswijk, C.; Post, J. N.; Heeren, R. M.; Karperien, M. Anal. Chem. 2015, 87, 3981-3988.

(14) Jiang, L.; Chughtai, K.; Purvine, S. O.; Bhujwalla, Z. M.; Raman, V.; Pasa-Tolic, L.; Heeren, R. M.; Glunde, K. Anal. Chem. 2015, 87, 5947-5956.

(15) Wu, S.; De Luca, F. J. Biol. Chem. 2004, 279, 4642-4647.

(16) Schmidt, K.; Hughes, C.; Chudek, J. A.; Goodyear, S. R.; Aspden, R. M.; Talbot, R.; Gundersen, T. E.; Blomhoff, R.; Henderson, C.; Wolf, C. R.; Tickle, C. Molecular and cellular biology 2009, 29, 2716-2729.

(17) Simonaro, C. M.; Sachot, S.; Ge, Y.; He, X.; Deangelis, V. A.; Eliyahu, E.; Leong, D. J.; Sun, H. B.; Mason, J. B.; Haskins, M. E.; Richardson, D. W.; Schuchman, E. H. PLoS One 2013, 8, e62715.

(18) Rocha, B.; Cillero-Pastor, B.; Eijkel, G.; Bruinen, A. L.; RuizRomero, C.; Heeren, R. M.; Blanco, F. J. Proteomics 2015, 15, 702713.

(19) Kosinska, M. K.; Liebisch, G.; Lochnit, G.; Wilhelm, J.; Klein, H.; Kaesser, U.; Lasczkowski, G.; Rickert, M.; Schmitz, G.; Steinmeyer, J. PLoS One 2014, 9, e91769.

(20) Cillero-Pastor, B.; Eijkel, G.; Kiss, A.; Blanco, F. J.; Heeren, R. M. Anal. Chem. 2012, 84, 8909-8916.

(21) Wittenauer, R.; Smith, L.; Aden, K. Osteoarthritis. Priority Medicines for Europe and the World: "A Public Health Approach to Innovation", Update on 2004 Background Paper, BP 6.12; World Health Organization, 2013.

(22) Blagojevic, M.; Jinks, C.; Jeffery, A.; Jordan, K. P. Osteoarthritis and cartilage/OARS, Osteoarthritis Research Society 2010, 18, 24-33.

(23) Sparvero, L. J.; Amoscato, A. A.; Dixon, C. E.; Long, J. B.; Kochanek, P. M.; Pitt, B. R.; Bayir, H.; Kagan, V. E. Chem. Phys. Lipids 2012, 165, 545-562.

(24) Pritzker, K. P.; Gay, S.; Jimenez, S. A.; Ostergaard, K.; Pelletier, J. P.; Revell, P. A.; Salter, D.; van den Berg, W. B. Osteoarthritis and cartilage/OARS, Osteoarthritis Research Society 2006, 14, 13-29.

(25) Hendriks, J.; Riesle, J.; Vanblitterswijk, C. A. Tissue Eng. 2006, 12, 2397-2405.
(26) Schindelin, J.; Rueden, C. T.; Hiner, M. C.; Eliceiri, K. W. Mol. Reprod. Dev. 2015, 82, 518-529.

(27) Sladkova, K.; Houska, J.; Havel, J. Rapid Commun. Mass Spectrom. 2009, 23, 3114-3118.

(28) Eijkel, G. B.; Kukrer Kaletas, B.; van der Wiel, I. M.; Kros, J. M.; Luider, T. M.; Heeren, R. M. A. Surf. Interface Anal. 2009, 41, 675685 .

(29) Ren, M.; Phoon, C. K. L.; Schlame, M. Prog. Lipid Res. 2014, 55, $1-16$.

(30) Schlame, M.; Greenberg, M. L. Biochim. Biophys. Acta, Mol. Cell Biol. Lipids 2017, 1862, 3-7.

(31) Paradies, G.; Paradies, V.; Ruggiero, F. M.; Petrosillo, G. Antioxid. Redox Signaling 2014, 20, 1925-1953.

(32) James, M. J.; Cleland, L. G.; Rofe, A. M.; Leslie, A. L. J. Rheumatol. 1990, 17, 521-527.

(33) Merrill, A. H., Jr.; Schmelz, E. M.; Dillehay, D. L.; Spiegel, S.; Shayman, J. A.; Schroeder, J. J.; Riley, R. T.; Voss, K. A.; Wang, E. Toxicol. Appl. Pharmacol. 1997, 142, 208-225.

(34) Chughtai, K.; Jiang, L.; Greenwood, T. R.; Glunde, K.; Heeren, R. M. J. Lipid Res. 2013, 54, 333-344.

(35) Veldhuizen, R.; Nag, K.; Orgeig, S.; Possmayer, F. Biochim. Biophys. Acta, Mol. Basis Dis. 1998, 1408, 90-108.

(36) Heywood, H. K.; Lee, D. A. J. Tissue Eng. Regen. Med. 2016, DOI: $10.1002 /$ term. 2126 .

(37) Bradley, R. M.; Stark, K. D.; Duncan, R. E. Mol. Nutr. Food Res. 2016, 60, 1804-1818.

(38) Le, C. H.; Mulligan, C. M.; Routh, M. A.; Bouma, G. J.; Frye, M. A.; Jeckel, K. M.; Sparagna, G. C.; Lynch, J. M.; Moore, R. L.; McCune, S. A.; Bristow, M.; Zarini, S.; Murphy, R. C.; Chicco, A. J. Circ.: Heart Failure 2014, 7, 172-183. 